\title{
Escala de Adição ao Facebook: estudos exploratórios de validade
}

\author{
Facebook Addiction Scale: exploratory validity studies \\ Escala de Adición a Facebook: estudios exploratorios de validez
}

DOI: $10.1590 / 1809-5844201824$

\begin{abstract}
José Pedro Cerdeira ${ }^{1}$
http://orcid.org/0000-0001-5487-6612

${ }^{1}$ (Instituto Politécnico de Coimbra, Escola Superior de Educação, Curso de Licenciatura em Comunicação Organizacional e Mestrado em Comunicação Organizacional - Cidadania, Confiança e Responsabilidade Social. Coimbra, Portugal)
\end{abstract}

\section{Resumo}

Este artigo apresenta os primeiros estudos de validade de uma versão portuguesa da Facebook Addiction Scale (AFS) de Koc e Gulyagci (2013). O estudo foi realizado com uma amostra de conveniência ( $\mathrm{N}=144)$, através de um questionário disponibilizado online. As análises estatísticas sobre os resultados obtidos sugerem que a escala apresenta excelentes propriedades psicométricas, semelhantes às da escala original: a análise das componentes principais, com rotação varimax, revela a existência de um só factor, capaz de explicar 63,7\% da variância total, enquanto a análise da consistência interna é de .91 (Alpha de Cronbach).

Palavras-chave: Escala de Adição ao Facebook. Dependências Comportamentais. Adições Comportamentais. Atitudes na Internet. Redes sociais.

\begin{abstract}
This paper intends to develop the first validity studies of a Portuguese version of Facebook Addiction Scale (AFS) by Koc and Gulyagci (2013). The study was carried out with a convenience sample ( $\mathrm{N}=144$ ), through a questionnaire made available online. Statistical analyzes of the results suggest that the scale has excellent psychometric properties, similar to those of the original scale. Analysis of the principal components, with varimax rotation, reveals the existence of a single factor, able of explaining $63,7 \%$ of the total variance, while the internal consistency analysis is .91 (Cronbach's alpha).

Keywords: Facebook Addiction Scale. Behavioral Dependencies. Behavioral Addiction. Internet attitudes. Social networks.
\end{abstract}

\section{Resumen}

Este artículo presenta los primeros estudios de validez de una versión portuguesa de Facebook Addiction Scale (AFS) de Koc y Gulyagci (2013). El estudio se efectuó con una muestra de conveniencia $(\mathrm{N}=144)$, a través de un cuestionario disponible en línea. Los análisis estadísticos 
sobre los resultados obtenidos sugieren que la escala presenta excelentes propiedades psicométricas, similares a las de la escala original: El análisis de los componentes principales, con rotación varimax, revela la existencia de un solo factor, capaz de explicar el 63.7\% de la varianza total, mientras que el análisis de la consistencia interna es de .91 (Alpha de Cronbach).

Palabras clave: Escala Adición Facebook. Depedencias Comportamentais. Adiciones de Comportamento a Facebook. Actitudes en Internet. Redes sociales.

\section{Introdução}

Na actualidade, a Internet é uma tecnologia amplamente difundida nas sociedades contemporâneas (OSBURG; LOHRMANN, 2017). As pessoas usam a Internet para atingirem objectivos (obtenção de conhecimentos, exercício de actividades profissionais, aquisição de produtos e serviços etc.) ou para satisfazerem necessidades psicológicas (reconhecimento, pertença, socialização, entretenimento etc.) (HEW, 2011, PEMPEK et al, 2009; PONTES et al, 2016; RYAN et al, 2014; WILSON et al, 2012; YANG; BROWN, 2013). Segundo a Internet World Stats (2017), entre 2000 e 2017, o índice de crescimento dos utilizadores da Internet foi de 936\%, atingindo os 3,7 mil milhões no corrente ano. Em 31 de março de 2017, um em cada dois habitantes utilizava a Internet, o que revela uma taxa de penetração de 49,7\% em relação à população mundial. Destes utilizadores, cerca de 637 milhões são europeus, dos quais sete milhões são portugueses (a taxa de penetração em Portugal é de 67,6\%, o que contabiliza 7.015.519 utilizadores) (MINIWATTS MARKETING GROUP, 2017). Quanto ao uso do Facebook à escala mundial, estatísticas de março de 2017, revelam que 1,28 mil milhões de pessoas usam diariamente o Facebook (FACEBOOK, 2017). Em Portugal, registam-se 5,6 milhões de subscritores, ou seja, 54\% dos portugueses são clientes do Facebook, o mesmo é dizer, 79,8\% dos utilizadores da Internet são clientes do Facebook ${ }^{1}$ (MINIWATTS MARKETING GROUP, 2017).

Face a estes valores, em conformidade com outras fontes (GUEDES et al, 2016; PONTES; GRIFFITHS, 2014), levantam-se várias questões. Uma delas refere-se ao quanto o uso genérico da Internet pode ou não ser entendido como uma adição (ou dependência) comportamental, com sintomas mais ou menos semelhantes aos sintomas das adições a substâncias (GRIFFITHS, 1996, 1999, 2000, GRIFFITHS; PONTES; KUSS, 2016; KUSS; LOPEZ-FERNANDEZ, 2016, KUSS; BILLIEUX, 2017; YOUNG, 1998). Uma outra, remete para alguns usos específicos da Internet, os quais poderão também ser concebidos como adições (ou dependências) comportamentais, por exemplo, aos videojogos e ao jogo online (GRIFFITHS et al, 2017, GRIFFITHS; PONTES, 2015, NG; WIEMER-HASTINGS, 2005), aos smartphones (KUSS, 2017; VAN DEURSEN et al, 2015) ou ao Facebook (RYAN et al, 2014; YOUNG et al, 2017).

1 Segundo a mesma fonte, na América do Sul estão registados cerca de 277 milhões de utilizadores da Internet, dos quais 139 milhões são brasileiros. Destes últimos, 111 milhões são subscritores do Facebook (30 jun. 2016) (MINIWATTS MARKETING GROUP, 2017). 
Nos diferentes casos, a investigação tem identificado alguns efeitos nefastos relacionados com estas adições ou dependências comportamentais. Por exemplo, a utilização excessiva do Facebook está associada à deterioração da qualidade das actividades diárias dos estudantes (distracções e procrastinação nas tarefas escolares mais frequentes), à vivência de estados de ansiedade e stress, de insónia e de depressão, a perturbações nos relacionamentos interpessoais, ao isolamento, à hostilidade nas relações com os outros, à deterioração das competências sociais, a alterações do humor etc. (EKIZOGLU; OZCINAR, 2011; ELGENDI, 2015; ELPHINSTON; NOLLER, 2011; GENCER; KOC, 2012; GRIFFITHS; PONTES, 2015; GUEDES et al, 2016; RYAN et al, 2014, 2016a, 2016b; VANNUCCI et al, 2017).

Face a este quadro é pertinente apelar à realização de mais investigação sobre as atitudes em relação à utilização das tecnologias de informação e comunicação na Internet, a qual pode envolver, por exemplo, a construção de novos instrumentos de avaliação das atitudes ou, em alternativa, passar pela tradução e adaptação de instrumentos já existentes, com a correspondente realização dos estudos de validade associados.

\section{Objectivo}

Este estudo tem o duplo objectivo de traduzir para português a Facebook Addiction Scale (Escala de Adição ao Facebook), construída por Koc e Gulyagci (2013), e de realizar alguns estudos exploratórios de avaliação das propriedades psicométricas da escala no sentido de dar resposta à escassez de trabalhos no campo de estudos das adições ou dependências comportamentais ao Facebook (PONTES; GRIFFITHS, 2014).

\section{Instrumento}

A Escala de Adição ao Facebook foi construída por Koc e Gulyagci (2013, p.280) a partir da “análise de trabalhos de investigação sobre as adições à Internet”. Segundo a análise realizada pelos autores, os oito itens que integram a escala estão relacionados com a avaliação dos sintomas cognitivos e comportamentais das adições, com a avaliação da saliência e do grau de conflito destes comportamentos com outras pessoas ou actividades, com as alterações do estado de humor e com a perda de controlo dos comportamentos, com as recaídas, as manifestações de abstinência e o restauro de comportamentos previamente eliminados. No essencial, a construção da escala pretendeu preencher uma lacuna na investigação sobre as novas dependências, já que eram escassos os instrumentos de reconhecimento dos sintomas de uma adição ou dependência às novas tecnologias de comunicação e poucos são ainda os estudos sobre a validade desses instrumentos. Nos anos mais recentes, com a vulgarização crescente do uso destas tecnologias, o tema das adições ganhou relevo, pelo que se justifica a realização de estudos exploratórios sobre o fenómeno, bem como a realização de estudos de validade sobre os instrumentos que o pretendem avaliar. 
Todos os itens da escala estão redigidos na afirmativa, pelo que os valores correspondentes às respostas podem ser somados para produzir um total. A adição ao Facebook será tão mais acentuada quanto mais elevado for o valor da pontuação resultante da soma das respostas aos oito itens da escala. Uma vez que a escala não tem sido muito usada em estudos de investigação, é pertinente realizar estudos exploratórios para determinar as propriedades psicométricas da escala e a sua validade.

\section{Amostra}

Foi usada uma amostra de conveniência ${ }^{2}$, constituída por 144 participantes voluntários, dos quais 46 são do sexo masculino (31,9\%) e 98 do sexo feminino $(68,1 \%)$, com idades compreendidas entre os 15 e os 35 anos $(M=21,94$, $D P=3,41)$. Os dados foram recolhidos através do GoogleDocs, com recurso a um questionário, durante os meses de abril-junho de 2016.

\section{Procedimentos}

A primeira parte do questionário inclui questões de caracterização da amostra: sexo, idade, ocupação, anos de exercício da actividade profissional, enquanto a segunda é composta pelos oito itens da Escala de Adição ao Facebook (KOC; GULYAGCI, 2013). As respostas foram codificadas numa escala de Likert de sete pontos, desde 1 - discordo fortemente até 7 - concordo fortemente. Todos os itens estão redigidos na afirmativa, pelo que a escala é aditiva, no sentido de uma maior pontuação corresponder à expressão de uma adição maior ao Facebook.

Para manter o mais possível o sentido original das frases da escala, foi seguida a sugestão de Hill e Hill (2008), ou seja, a escala foi traduzida para português e posteriormente retraduzida para inglês por um outro investigador, tendo-se procedido depois à análise e discussão das diferenças nas duas versões. A versão final foi avaliada por um grupo de cinco estudantes de licenciatura, para identificar eventuais problemas de compreensão do sentido dos itens e evitar, assim, possíveis mal-entendidos. Nesta fase, procedeu-se a uma alteração do sistema de codificação das respostas, passando para uma escala Likert de sete

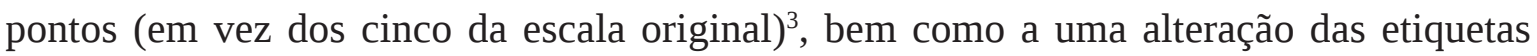
associadas, as quais passaram para 1-discordo fortemente a 7-concordo fortemente, em vez

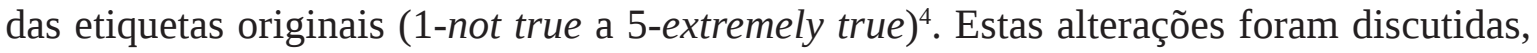
tendo sido unânime o entendimento de que produziam um ganho de clareza.

2 O critério de formação da amostra foi a conveniência, o que significa que se trata de uma amostra não probabilística porque os sujeitos foram seleccionados apenas em função da acessibilidade e da sua disponibilidade para responderem ao questionário.

3 As escalas de Likert são um dos procedimentos mais usados para codificar as respostas dos sujeitos num estudo de opinião. No caso deste estudo exploratório, o formato das respostas foi alargado para sete níveis para aumentar a amplitude das respostas possíveis.

4 As etiquetas originais ("Não é verdade" e "Extremamente verdadeiro") foram substituídas por etiquetas denotando maior ou menor concordância com o sentido das afirmações. 
Os dados foram analisados com recurso ao Statiscal Package for Social Sciences (SPSS, versão 21) ${ }^{5}$. Foram eliminados nove sujeitos da amostra (5,9\%) por terem respondido apenas às perguntas de caracterização da primeira parte do questionário. Não se detectaram não-respostas.

\section{Resultados}

Na Tabela 1, apresentam-se os valores da média e do desvio-padrão dos itens da escala. A média total da escala foi de 21,77 ( $\mathrm{DP}=10.88$ ), não se detectaram diferenças estatisticamente significativas em função do sexo com o teste $t$ de student. Contudo, fazendo uma análise por item foram identificadas diferenças estatisticamente significativas no item 4 (O uso que faço do Facebook interfere com as minhas actividades sociais), com um valor de $t(142)=2,148, \mathrm{p}=.033$. Os participantes do sexo masculino apresentam uma média mais alta $(M=3,15, D P=1,59)$ por comparação como os do sexo feminino $(M=2,54, D P=1,59)$. A respeito das restantes variáveis sociodemográficas da amostra, não foram detectadas diferenças estatisticamente significativas nos valores das médias.

Tabela 1: Valores da média, do desvio-padrão e correlações inter-itens da Escala de Adição ao Facebook $(\mathrm{N}=144)$

\begin{tabular}{ccccccccccc}
\hline $\begin{array}{c}\text { Itens da } \\
\text { escala }\end{array}$ & $\mathbf{M}$ & $\mathbf{D P}$ & $\mathbf{1}$ & $\mathbf{2}$ & $\mathbf{3}$ & $\mathbf{4}$ & $\mathbf{5}$ & $\mathbf{6}$ & $\mathbf{7}$ & $\mathbf{8}$ \\
\hline 1. & 3.18 & 1.68 & - & & & & & & \\
2. & 3.15 & 1.97 & .56 & - & & & & & \\
3. & 2.75 & 1.78 & .68 & .51 & - & & & & \\
4. & 2.74 & 1.61 & .52 & .49 & .61 & - & & & \\
5. & 2.58 & 1.76 & .53 & .47 & .57 & .62 & - & & \\
6. & 2.72 & 1.86 & .51 & .50 & .59 & .61 & .63 & - & \\
7. & 2.31 & 1.49 & .55 & .51 & .67 & .55 & .61 & .72 & - & \\
8. & 2.34 & 1.53 & .55 & .45 & .65 & .55 & .68 & .70 & .75 & - \\
\hline
\end{tabular}

Fonte: Elaborado pelo autor.

A correlação inter-itens mais baixa é de .45 (entre o item 2 e o item 8), a mais alta de .75 (entre os itens 7 e 8), com uma média de correlações de .58. Todos os valores de correlação inter-itens são significativos $(\mathrm{p}<.01)$. O determinante da matriz de correlações é de .006, o que indica a possibilidade de se realizar uma análise factorial, possibilidade

$\overline{5}$ O SPSS é um programa informático para análise estatística de dados, muito usado nos estudos em Ciências Sociais. 
essa que é sugerida também pelo Teste de Esfericidade de Bartlett $\left(x^{2}(28, N=144)=707.191\right.$ $(p<.000))$ e pelo valor do índice de Kaiser-Meyer-Olkin (K.M.O.=.913) ${ }^{6}$.

Tabela 2: Valores das correlações item-total corrigido, do quadrado da correlação múltipla e do alpha (se o item for removido) dos itens da Escala de Adição ao Facebook (N=144)

\begin{tabular}{cccc} 
Itens da escala & $\begin{array}{c}\text { Correlação corrigida } \\
\text { item-total }\end{array}$ & $R^{2}$ & $\begin{array}{c}\text { alpha } \\
\text { (Se o item for removido) }\end{array}$ \\
\hline 1. & .69 & .54 & .91 \\
2. & .61 & .41 & .92 \\
3. & .76 & .63 & .90 \\
4. & .71 & .53 & .91 \\
5. & .73 & .57 & .90 \\
6. & .76 & .63 & .90 \\
7. & .78 & .67 & .90 \\
8. & .77 & .67 & .90 \\
\hline
\end{tabular}

Fonte: Elaborado pelo autor.

Verificadas as condições para a factorialização da matriz de correlações, usa-se o método das componentes principais, com rotação varimax, para uma análise factorial exploratória ${ }^{7}$. Esta análise produziu uma solução com uma única componente com valores próprios superiores à unidade $(5,10)$, capaz de explicar uma porção bastante grande da variabilidade geral das respostas dadas à totalidade dos itens da escala (63,8\%). Os índices de saturação dos oito itens são todos superiores a .69 (Tabela 2).

O valor alpha de Cronbach $^{8}$ é de .91, o que sugere uma elevada consistência interna dos itens da escala, a qual é confirmada pela análise dos valores do quadrado da correlação múltipla, que variam entre .41 e.67, o que sugere que os itens partilham entre si percentagens importantes da sua variância (Tabela 3). O valor alpha baixa com a remoção de qualquer um dos itens da escala, excepto no caso do item 2 (A primeira coisa de que me lembro quando acordo é ligar-me ao Facebook), cuja remoção mantém o valor do alpha constante.

6 O Teste de Esfericidade de Bartlett e índice de Kaiser-Meyer-Olkin são duas medidas que permitem avaliar as condições requeridas para fazer uma análise factorial de dados (MARÔCO, 2018).

7 O método das componentes principais corresponde a um método estatístico de análise de dados, usado para converter um conjunto de observações de variáveis não correlacionadas em valores com significado estatístico (MARÔCO, 2018).

8 O alpha Cronbach é um indicador do grau de consistência interna de uma medida psicológica. 
Tabela 3: Saturações factoriais pelo método das componentes principais, comunalidades, valores próprios e percentagem da variância explicada dos itens da Escala de Adição ao Facebook $(N=144)$

\begin{tabular}{lcc}
\hline & Componente & $h^{2}$ \\
& $\mathbf{1}$ & .72 \\
\hline 07_Sinto-me ansioso quando não consigo aceder ao Facebook. & .85 & .70 \\
08_Já tentei gastar menos tempo no Facebook, mas não consegui. & .84 & .69 \\
06_A minha família e os meus amigos acham que eu gasto muito tempo & .83 & \\
Com o Facebook. & .63 \\
03_Perco horas de sono por passar muito tempo no Facebook. & .83 \\
05_Quando estou em baixo, ligo-me ao Facebook para me sentir & .80 \\
melhor. & & .64 \\
04_O uso que faço do Facebook interfere com as minhas actividades & .78 \\
sociais. & & .60 \\
01_Tenho dificuldades em concentrar-me no meu trabalho académico & .76 \\
por causa do uso que faço do Facebook. & & .58 \\
02_A primeira coisa de que me lembro quando acordo é ligar-me ao & .69 \\
Facebook. & & .48 \\
\hline Valores próprios & 5.10 \\
\% da variância explicada & 63.7 \\
\hline
\end{tabular}

Fonte: Elaborado pelo autor.

No seu conjunto, estes resultados sugerem que os oito itens da adaptação portuguesa da Escala de Adição ao Facebook de Koc e Gulyagci (2013) medem uma só e mesma dimensão, com uma excelente consistência interna.

\section{Conclusões}

O principal objectivo foi o de desenvolver os estudos exploratórios de validade de constructo de uma versão portuguesa da Escala de Adição ao Facebook de Koc e Gulyagci (2013), no sentido de disponibilizar um meio para a investigação do fenómeno da utilização de uma das ferramentas tecnológicas mais vulgarizadas: o Facebook. Apesar de se tratar de um primeiro estudo, com uma amostra de conveniência limitada (o qual não exclui a necessidade de se realizarem estudos complementares de validade), não deixa de ser pertinente realçar alguns dos resultados obtidos. Em primeiro lugar, há a destacar a estrutura uni-factorial da escala. A utilização do método das componentes principais, com rotação varimax, permitiu a produção de uma solução com uma só componente principal, capaz de explicar 63,7\% da variância total e na qual a totalidade dos itens satura significativamente acima de .69 - com valores de comunalidade bastante importantes (acima de .48), o que 
parece reforçar o modelo factorial original dos autores da escala. Em segundo lugar, o facto do valor alpha encontrado ser muito alto (.91), associado à circunstância de a remoção de qualquer um dos itens da escala contribuir para uma redução desse valor, sugere uma consistência interna muito boa.

Uma vez que foi usada uma amostra de conveniência, constituída por sujeitos relativamente heterogéneos, as conclusões são limitadas à natureza da amostra, pelo que não é possível sugerir qualquer tipo de associação entre os resultados obtidos com a administração da escala e eventuais comportamentos de adição ou de dependência em relação ao Facebook. Para esse propósito, são necessários mais estudos.

\section{Referências}

EKIZOGLU, N.; OZCINAR, Z. A study of developing an anxiety scale towards the internet. Procedia Social and Behavioral Sciences, v.15, p.3902-3911, 2011.

ELGENDI, H. The effect of Facebook on college student's. International Journal of Networks and Communications, v.5, n.2, p.37-40, 2015.

ELPHINSTON, R. A.; NOLLER, P. Time to face it! Facebook intrusion and the implications for romantic jealousy and relationship satisfaction. Cyberpsychology, Behavior, and Social Networking, v.14, n.11, p.631-635, 2011.

FACEBOOK. Facebook newsroom: company info. 2017. Available at: http://newsroom. fb.com/company-info/. Accessed on: 20 jun. 2017.

GENCER, S. L.; KOC, M. Internet abuse among teenagers and its relations to internet usage patterns and demographics. Educational Technology \& Society, v.15, n.2, p.25-36, 2012.

GRIFFITHS, M. D. Behavioural addiction: an issue for everybody? Employee Councelling Today: the journal of workplace learning, v.8, n.3, p.19-25, 1996.

. Internet addiction: fact or fiction? Psychologist, v.12, p.246-250, 1999.

Does Internet and computer 'addiction' exist? Some case-study evidence. CyberPsychology \& Behavior, v.3, n.2, p.211-218, 2000.

.; PONTES, H. M. Addiction and entertainment products. In: NAKATSU, R.; RAUTERBERG, M.; CIANCARINI, P. (Eds.). Handbook of digital games and entertainment technologies. Singapore: Springer, 2015, p.1-22.

.; KUSS, D. J.; GORTARI, A. B. Videogames as therapy: an updated selective review of the medical and psychological literature. International Journal of Privacy and Health Information Management, v.5, n.2, p.71-96, 2017.

.; PONTES, H. M.; KUSS, D. J. Online addictions: Conceptualizations, debates and controversies. Addicta: the turkish journal on addictions, v.3, n.2, p.151-164, 2016.

GUEDES, E.; SANCASSIANI, F.; CARTA, M. G.; CAMPOS, C.; MACHADO, S.; KING, A. L., NARDI, A. E. Internet addiction and excessive social networks use: what about Facebook? Clinical Pratice \& Epidemology in Mental Health, v.12, p.43-48, 2016.

HEW, K. F. Students and teachers use of Facebook. Computers in Human Behavior, v.27, n.2, p.662676, 2011. 
HILL, M. M.; HILL, A. Investigação por questionário. Lisboa: Edições Sílabo, 2008.

KOC, M.; GULYAGCI, S. Facebook addiction among turkish college students: The role of psychological health, demographic, and usage characteristics. Cyberpsychology, Behavior, and Social Networking, v.16, n.4, p.279-284, 2013.

KUSS, D. J. OP-58: Problematic mobile phone use and mobile phone addiction. Journal of Behavioral Addictions, v.6, n.1, p.28-29, 2017.

KUSS, D. J.; BILLIEUX, J. Technological addictions: conceptualisation, measurement, etiology and treatment. Addictive behaviors, v.64, p.231-233, 2017.

KUSS, D. J.; LOPEZ-FERNANDEZ, O. Internet addiction and problematic internet use: a systematic review of clinical research. World Journal of Psychiatry, v.6, n.1, p.143-176, 2016.

MARÔCO, J. Análise estatística com o SPSS Statistics. Lisboa: Edições Sílabo, 2018.

MINIWATTS MARKETING GROUP. World internet usage statistics news and population stats. 2017. Available at: www.internetworldstats.com/stats.htm. Accessed on: 2 jun. 2017.

NG, B. D.; WIEMER-HASTINGS, P. Addiction to the internet and online gaming. CyberPsychology \& Behavior, v.8, n.2, p.110-113, 2005.

OSBURG, T., \& LOHRMANN, C. (Eds.). Sustainability in a digital world. New oportunities though new technologies. Cham: Springer International Publishing, 2017.

PEMPEK, T. A.; YERMOLAYEVA, Y. A.; CALVERT, S. L. College students social networking experiences on Facebook. Journal of Applied Developmental Psychology, v.30, n.3, p.227-238, 2009.

.; GRIFFITHS, M. D. A dependência à internet no contexto português: mito, ficção ou realidade? In: IX ${ }^{\circ}$ CONGRESSO IBEROAMERICANO DE PSICOLOGIA e II ${ }^{\circ}$ CONGRESSO DA ORDEM DOS PSICÓlOGOS PORTUGUESES. Setembro 2014. Actas. Available at DOI: 10.13140/2.1.1608.3522. Accessed on: 20 jun. 2017.

PONTES, H. M.; ANDREASSEN, C. S.; GRIFFITHS, M. D. Portuguese validation of the Bergen Facebook Addiction Scale: an empirical study. International Journal of Mental Health Addiction, v.14, p.1062-1073, 2016.

RYAN, T.; CHESTER, A.; REECE, J.; XENOS, S. The uses and abuses of Facebook: a review of Facebook addiction. Journal of Behavioral Addictions, v.3, n.3, p.133-148, 2014.

; CHESTER, A.; REECE, J.; XENOS, S. A qualitative exploration of Facebook addiction: working toward construct validity. Addicta: The Turkish Journal on Addictions, v.3, n.1, p.55-76, $2016 \mathrm{a}$.

.; REECE, J.; CHESTER, A.; XENOS, S. Who gets hooked on Facebook? An exploratory typology of problematic Facebook users. Cyberpsychology: journal of psychosocial research on cyberspace, v.10, n.3, p.1-25, 2016b.

VAN DEURSEN, A. J.; BOLLE, C. L.; HEGNER, S. M.; KOMMERS, P. A. Modeling habitual and addictive smartphone behavior: the role of smartphone usage types, emotional intelligence, social stress, self-regulation, age, and gender. Computers in Human Behavior, v.45, p.411-420, 2015.

VANNUCCI, A.; FLANNERY, K. M.; OHANNESSIAN, C. M. Social media use and anxiety in emerging adults. Journal of Affective Disorders, v.207, p.163-166, 2017.

WILSON, R. E.; GOSLING, S. D.; GRAHAN, L. T. A review of Facebook research in the social sciences. Perspectives on Psychological Science, v.7, n.3, p.203-220, 2012.

YANG, C. C.; BROWN, B. B. Motives for using Facebook, patterns of Facebook activities and late adolescents social adjustment to college. Journal of Youth and Adolescence, v.42, n.3, p.403-416, 2013. 
YOUNG, K. S. Internet addiction: the emergence of a new clinical disorder. CyberPsychology \& Behavior, v.1, n.3, p.237-244, 1998.

YOUNG, N. L.; KUSS, D. J.; GRIFFITHS, M. D., HOWARD, C. J. Passive Facebook use, Facebook addiction, and associations with escapism: an experimental vignette study. Computers in Human Behavior, v.71, p.2431, 2017.

\section{José Pedro Cerdeira}

Doutorado em Psicologia Social na Universidade de Coimbra. Professor Coordenador. Director do curso de licenciatura ( $1^{\circ}$ ciclo Bolonha) em Comunicação Organizacional e do curso de mestrado em Comunicação Organizacional - Cidadania, Confiança e Responsabilidade Social ( $2^{\circ}$ ciclo Bolonha). Coordenador da área científica de Ciências da Comunicação, das Organizações e dos Media (CCOM) e membro do Centro de Estudos de Psicologia Social (CEPS), Escola Superior Educação, Instituto Politécnico de Coimbra. E-mail: jpcerd@esec.pt.

Recebido em: 28.07.2017

Aprovado em: 16.07.2018

Este artigo é publicado em acesso aberto (Open Access) sob a licença Creative Commons Attribution Non-Commercial (CC-BY-NC), que permite uso, distribuição e reprodução em qualquer meio, sem restrições, desde que sem fins comerciais e que o trabalho original seja corretamente citado. 